NASA/TM-2004-213201

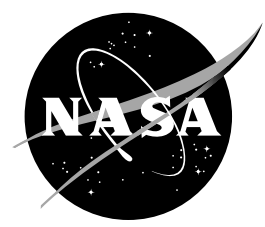

Neural Network for Image-to-Image Control of Optical Tweezers

Arthur J. Decker, Robert C. Anderson, Kenneth E. Weiland, and Susan Y. Wrbanek Glenn Research Center, Cleveland, Ohio

Restrict from public Web access based on NASA Internet Publishing Content Guidelines, November 15, 2001. 
Since its founding, NASA has been dedicated to the advancement of aeronautics and space science. The NASA Scientific and Technical Information (STI) Program Office plays a key part in helping NASA maintain this important role.

The NASA STI Program Office is operated by Langley Research Center, the Lead Center for NASA's scientific and technical information. The NASA STI Program Office provides access to the NASA STI Database, the largest collection of aeronautical and space science STI in the world. The Program Office is also NASA's institutional mechanism for disseminating the results of its research and development activities. These results are published by NASA in the NASA STI Report Series, which includes the following report types:

- $\quad$ TECHNICAL PUBLICATION. Reports of completed research or a major significant phase of research that present the results of NASA programs and include extensive data or theoretical analysis. Includes compilations of significant scientific and technical data and information deemed to be of continuing reference value. NASA's counterpart of peerreviewed formal professional papers but has less stringent limitations on manuscript length and extent of graphic presentations.

- TECHNICAL MEMORANDUM. Scientific and technical findings that are preliminary or of specialized interest, e.g., quick release reports, working papers, and bibliographies that contain minimal annotation. Does not contain extensive analysis.

- CONTRACTOR REPORT. Scientific and technical findings by NASA-sponsored contractors and grantees.
- CONFERENCE PUBLICATION. Collected papers from scientific and technical conferences, symposia, seminars, or other meetings sponsored or cosponsored by NASA.

- SPECIAL PUBLICATION. Scientific, technical, or historical information from NASA programs, projects, and missions, often concerned with subjects having substantial public interest.

- TECHNICAL TRANSLATION. Englishlanguage translations of foreign scientific and technical material pertinent to NASA's mission.

Specialized services that complement the STI Program Office's diverse offerings include creating custom thesauri, building customized databases, organizing and publishing research results ... even providing videos.

For more information about the NASA STI Program Office, see the following:

- Access the NASA STI Program Home Page at http://www.sti.nasa.gov

- E-mail your question via the Internet to help@sti.nasa.gov

- Fax your question to the NASA Access Help Desk at 301-621-0134

- Telephone the NASA Access Help Desk at 301-621-0390

- Write to:

NASA Access Help Desk

NASA Center for AeroSpace Information 7121 Standard Drive

Hanover, MD 21076 
NASA/TM-2004-213201

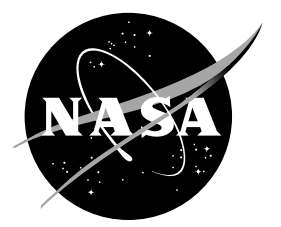

\section{Neural Network for Image-to-Image Control of Optical Tweezers}

Arthur J. Decker, Robert C. Anderson, Kenneth E. Weiland, and Susan Y. Wrbanek Glenn Research Center, Cleveland, Ohio

Restrict from public Web access based on NASA Internet Publishing Content Guidelines, November 15, 2001.

Prepared for the

2004 Annual Meeting

sponsored by the International Society for Optical Engineering

Denver, Colorado, August 2-6, 2004

National Aeronautics and

Space Administration

Glenn Research Center 
This report is a formal draft or working paper, intended to solicit comments and ideas from a technical peer group.

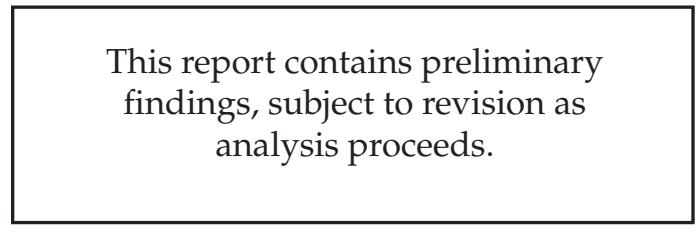

This work was sponsored by the Low Emissions Alternative Power Project of the Vehicle Systems Program at the NASA Glenn Research Center.

Available from

NASA Center for Aerospace Information 7121 Standard Drive

Hanover, MD 21076
National Technical Information Service 5285 Port Royal Road Springfield, VA 22100 


\title{
Neural Network for Image-to-Image Control of Optical Tweezers
}

\author{
Arthur J. Decker, Robert C. Anderson, Kenneth E. Weiland, and Susan Y. Wrbanek \\ National Aeronautics and Space Administration \\ Glenn Research Center \\ Cleveland, Ohio 44135
}

\begin{abstract}
A method is discussed for using neural networks to control optical tweezers. Neural-net outputs are combined with scaling and tiling to generate 480X480-pixel control patterns for a spatial light modulator (SLM). The SLM can be combined in various ways with a microscope to create movable tweezers traps with controllable profiles. The neural nets are intended to respond to scattered light from carbon and silicon carbide nanotube sensors. The nanotube sensors are to be held by the traps for manipulation and calibration. Scaling and tiling allow the 100X100-pixel maximum resolution of the neural-net software to be applied in stages to exploit the full 480X480-pixel resolution of the SLM. One of these stages is intended to create sensitive null detectors for detecting variations in the scattered light from the nanotube sensors.
\end{abstract}

\section{INTRODUCTION}

Optical tweezers are being developed at NASA Glenn to hold and to manipulate micro-scale and nano-scale sensors. Two NASA projects, among many, that currently support nanotechnology, in general, and the development of nanotube sensors, specifically, are Alternate Energy Foundation Technologies and the Independent Research and Development Fund. One objective is to manipulate and calibrate nanotube sensors that range in size from micrometers, the initial objective, to the order of nanometers, eventually. The sensors are to be made from carbon and silicon carbide. Some proposed tasks include using tweezers to manipulate the sensors to straddle electrodes and to hold isolated sensors for calibration. Calibration is to be accomplished by detecting light scattered from isolated sensors or from multiple interacting particles. Single and multiple tweezers traps can be generated and profiled using phase modulators ${ }^{1}$ such as spatial light modulators (SLM). The traps can be created using an isolated SLM, a SLM as a component of an interferometer, or a SLM together with other trap-generating components. ${ }^{2-4}$ A SLM can be light-patternprogrammed to move the traps, to change the angular momentum of a trap beam, or to change the intensity profile of a trap.

One objective of the NASA Glenn work in nano technology is the fast detection and interpretation of scattered-light patterns from one or more tweezers traps. Detection and interpretation of the scattered light are expected to be performed in potentially noisy environments. The environment may include, for example, the electrodes and structures inserted by the sensor developers, light detection channels, unused sensors, unused tool particles and other residue. The interpretation of the scattered light is to be used for sensor calibration or the generation of SLM control patterns for manipulating the traps and the trapped sensors. Artificial neural networks are discussed in this paper for the fast mapping of scattered-light patterns into SLM control patterns.

Artificial neural networks have proven to be sensitive detectors of optical pattern changes buried in noise, ${ }^{5,6}$ and can be used to perform fast pattern-to-pattern transformations. ${ }^{7,8}$ The SLM, used for trap generation and profiling, is essentially just an optical-pattern-controlled device. A 480X480 pixel intensity pattern is transmitted to the SLM. The SLM, in turn, converts the intensity pattern into a phase pattern to be modulated onto an expanded laser beam reflected from the SLM. The phase profile, generated by the SLM, is then transferred to the input of a microscope that produces the optical traps for holding and manipulating the nanotube sensors. The objective is to train a neural net to transform a 480X480 pixel pattern of scattered light from a trap array into a 480X480 pixel control pattern for the SLM. Other objectives are to generate outputs 
representative of the scattered light for calibration and to perform SLM pattern-to-pattern transformations for a neural-net sequencer.

Unfortunately, achievement of the simply stated objective is not so straightforward, but previous results on the use of neural nets for holographic non-destructive-evaluation (NDE) ${ }^{5,6,9}$ have transferred well to the tweezers control application. One problem was that the neural-net software and computers used for this study were restricted to about 10,000 inputs or outputs (100X100 pixels rather than 480X480 pixels). Hence, image-data compression of some kind was required. An array of 48X48 optical channels of data was selected for the discussions of this paper. The training set size required for noisy optical data increases as the square of the number of inputs, so that it is desirable to work with small input arrays. A more difficult problem in adapting neural-net NDE rules is to handle multiple training classes and noise sources. A training class is a set of input patterns that elicit the same output from the neural network. ${ }^{9}$ Multiple noise-corrupted samples of the members of a class must be presented to the net during training. Each training class has proven to require about three hidden-layer nodes, when a feed-forward neural net is trained. The required number of hidden-layer nodes is not affected by a noise source, but enough noise samples are required to cover the noise distribution function. Randomly selected noise patterns, equal in number to ten percent of the number of inputs, suffice. Hence, the storage requirements for one class in a training set increase in proportion to the square of the number of inputs multiplied by the number of members in the class and the number of independent noise sources. Both additive and multiplicative noise sources may be important. Large training requirements make neural nets impractical for many applications, but nets are feasible where the number of classes and the input size can be limited. The tweezers control application, in particular, has some important features that can be exploited by the neural-net approach.

This paper will discuss the feasibility of using neural nets to interpret optical-tweezers scattered-light patterns and generate SLM control patterns. The neural nets are intended to perform pattern-to-pattern and pattern-to-feature mappings. Ideally, a neural net is to be trained with an experimental training set that incorporates noise sources and class characteristics, some possibly unknown to the net designer. An experimental test set can be used for calibration of the net, once the net is trained. ${ }^{10}$ But the feasibility of using the neural-net approach must be determined using model generated data. The model does not have to be exact, but it must generate data with the same order of complexity as the experimental data. A training set can then be assembled according to the rules and references just mentioned. The optical tweezers application is very well suited for this approach. SLM control patterns are calculated patterns, applied to the SLM through the VGA port of a computer. A software package that displays, or calculates and displays, images can be used to apply patterns to the SLM. ${ }^{11}$ The simplest model for tweezers applications treats the SLM and microscope-image planes as Fourier conjugates. A laser beam is expanded by a telescope, and the expanded beam is reflected from the SLM. The reflected beam is multiplied by a phase factor generated by the SLM in response to the applied calculated pattern. A telescope, microscope-objective combination then scales and Fourier transforms the SLM output to create one or more tweezers traps. The SLM shown in Fig. 1 has been used for phase modulation in a reflecting mode.

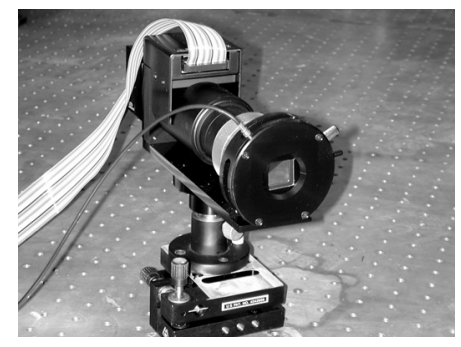

Fig. 1. Reflecting SLM with $480 \times 480$ programmable

The process for calculating the Fourier conjugates to assemble the training sets, where one conjugate is converted to an arbitrary intensity distribution of tweezers traps, is described very well in a pair of references. ${ }^{1,12}$ The references provide algorithms for transforming optimally the trap intensity profiles into the phase factors to be generated by the SLM. The pattern-programmed SLM acts as a phase-only hologram called a kinoform. ${ }^{13}$ The phase distributions are calculated modulo $2 \pi$. The SLM, in fact, can transform the phase of the laser beam in a range slightly greater than 0 to $2 \pi^{2}$ Figure 2 shows a phase distribution and a

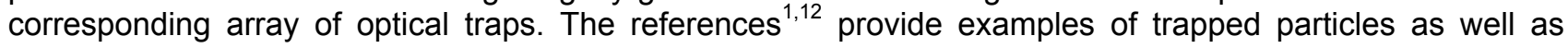
phase modulations for changing the focus and angular momentum of a trap beam. 

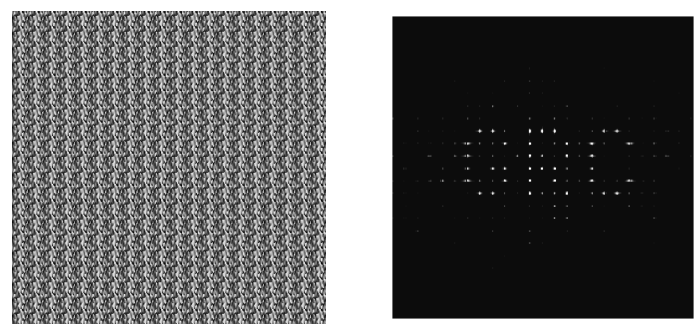

Fig. 2. Tiled phase distribution and trap array.

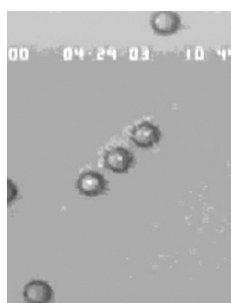

Fig. 3. Linear array of trapped micro particles.

The properties of the Discrete Fourier Transform (DFT) implemented by the Fast Fourier Transform (FFT) algorithms will be relied upon to generate the training sets. In fact, that model for evaluating the performance of the neural nets is really highly simplified. The traps are affected by aberrations. Calculations of exact scattering profiles, let alone trap forces and moments, are very complex, particularly for non-spherical particles. Currently, the tweezers models are being updated to include exact scattering effects in the presence of aberrations. ${ }^{14,15}$ Phase distributions needed to modify the effects of aberrations, for example, can be added to the SLM phase distribution $(\bmod 2 \pi)$. A neural net can be trained and calibrated, in principle, as a very sensitive null detector of the difference between a desired (or postulated) light profile and the measured profile. $^{10}$ This feature applies to patterns buried in noise. The output of this kind of neural net might be used separately as part of a feedback control loop to cancel aberrations or other undesired effects. This particular property is one of the major motivations for developing neural nets for tweezers control.

The Fourier transform relationship between the SLM plane and the tweezers focal plane is very useful for compressing the number of inputs to the neural network as discussed in the next section.

\section{TILING AND SCALING NEURAL-NET INPUTS AND OUTPUTS}

The 480X480 pixel patterns must be compressed to no more than 10,000 (96X96) pixels for neuralnet processing. The neural-net outputs must be increased again to $480 \times 480$ pixels, in order to use fully the resolution of the SLM.

\subsection{Stages of processing}

Neural-net processing can be accomplished in stages, with separate nets for each stage. The first stage requires zoomed-down or de-magnified images. The image is first separated into tiles. The $480 \times 480$ pixel array might be separated into 100 tiles containing 48X48 pixels each. A single neural-net input is drawn from each tile. This process can be performed with or without filtering. Without filtering, a single pixel is recorded from each tile. Fixed or randomly selected positions can be selected within a tile. Random selection of positions has been effective for neural-net processing of speckled fringe patterns. Or the data can be rebinned by averaging adjacent pixels using a weighting function or filter. The process selected will depend on the intent of the experimenters as well as the density and the nature of the sensors. Micro particles, or the images of smaller particles, might cover several pixels as is shown for the trapped and free particles in Fig. $3 .^{3}$ The objective of the first stage of processing is to train the net to generate a kinoform to move a tweezers beam to a tile containing a sensor or other significant particle. Particle identification is to be performed in the presence of noise.

The $48 \times 48$ pixel holograms generated by the neural-network are easily converted to $480 \times 480$ holograms by re-tiling. The kinoform phase pattern in Fig. 2 was actually assembled by re-tiling $48 X 48$ pixel patterns. A 48X48 pixel "GRC" was transformed to a 48X48 pixel phase pattern using the procedures from the references. ${ }^{1,12}$ The computed patterns were then tiled to produce the $480 \times 480$ pixel results shown in the figure. The computed or net-generated DFT is periodic. Hence, the effect of tiling is simply to increase the diameter of the ray pencil forming an optical trap and to increase the resolution in the imaging plane. The 
same arguments hold for 96X96 pixel holograms or for 12X12 pixel holograms. The choices lead to smaller or larger inter-pixel distances, respectively, in the 480X480 trap array.

The second and third stages are similar in their neural-net requirements and processing. For the second stage, the imaging system zooms in on the tile selected in the first stage. A second neural net is trained to generate holograms to fine-position the tweezers beam within the zoomed region. A $12 \times 12$ hologram might be selected during this stage. The hologram is then scaled rather than tiled so that the second-stage-hologram control continues to be confined to a $12 \times 12$ pixel region. The scaled hologram is simply added $(\bmod 2 \pi)$ to the hologram generated during the first stage. The summed holograms then move the tweezers trap to the correct pixel position in the 480X480 array. A complication is that the phase patterns are wrapped. This requires that the selected scaling procedure should not increase the number of pixels across a discontinuity in the phase map. Doing so does not completely obliterate a trap, but it does reduce the laser power directed to the trap. Figure 4 shows a one-dimensional example. In Fig. 4a, there are two onepixel trap locations along a 48-pixel axis. A cut through a corresponding hologram is also shown. The computed efficiency of the hologram is about 81 percent for light entering the desired traps. Figure $4 \mathrm{~b}$ shows the hologram scaled to 480 pixels and the corresponding traps. The traps appear at the same coordinate numbers (as they should for a scaled hologram but not a tiled hologram), and the computed efficiency was again about 81 percent.

The third stage uses scaled holograms to modify trap shapes. The total phase variation of a hologram might not exceed $2 \pi$. The eventual objective is to use the neural net as a sensitive detector of differences in the measured scattered light from trapped particles when the trap shape is varied. Various processes, linear, non-linear and fluorescent, might be involved in scattering. Neural nets have been trained and calibrated as sensitive null detectors of optical pattern changes. ${ }^{10}$ The nets can be trained to ignore noise effects, and can be trained and calibrated with model-generated or experimental data. Hence the nets can be used to compare model predictions with measured scattering. A possible application is to train a net to generate holograms to compensate for aberrations. The compensating holograms are to be added $(\bmod 2 \pi)$ to the holograms from the first and second stages.

(a)

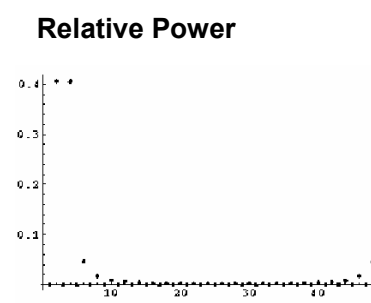

Pixel Number
Phase (rad.)

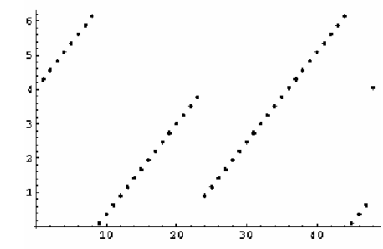

Pixel Number

(b)
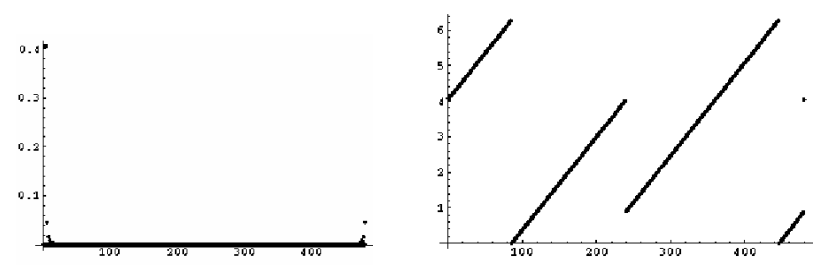

Fig. 4. (a) Traps and hologram at 48 pixel resolution. (b)Traps and hologram after scaling to 480 pixels.

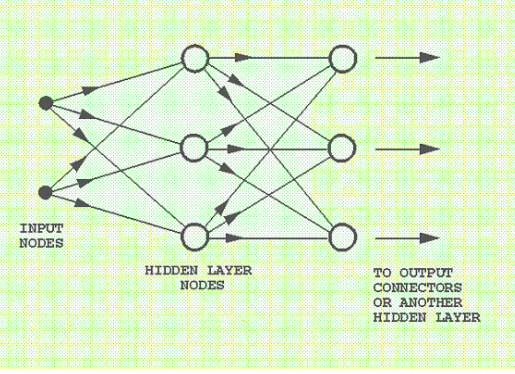

Fig. 5. Schematic of feed-forward net. 


\subsection{Consequence of multi-stage neural-net processing}

The multi-stage approach allows the net resource requirements to be controlled. This paper discusses results for $48 \times 48$ pixel-input nets for the first stage and $12 \times 12$ pixel-input nets for the second stage. The size of the nets for the third stage will depend on the specific models and aberration conditions, but are expected to range from $12 \times 12$ pixels to $48 \times 48$ pixels. The limits of the third stage are demonstrated using $48 \times 48$ phase patterns as inputs and 196 class indices as outputs.

All the artificial neural networks used for this work were feed-forward nets with one hidden layer as shown schematically in Fig. 5 . These nets are trained using versions of the back-propagation algorithm ${ }^{16}$, and are particularly well suited for handling noisy data. The rules for training set and net design have been tested extensively for the processing of speckled fringe patterns and appear to apply well to the tweezers applications.

The first application is the handling of first-stage tweezers data, where the tweezers image plane is compressed to $48 \mathrm{X} 48$ pixels with or without filtering. The objective is to train the net to generate holograms which direct the tweezers beam to a tile containing a particle or sensor. Several features of neural net training and performance are demonstrated next to achieve this objective.

\section{NEURAL-NET REQUIREMENTS FOR FIRST-STAGE PROCESSING}

\subsection{Coordinates and training records}

Pixels are to be labeled from the lower left beginning at $(0,0)$ for the $48 \times 48$ array. The origin of coordinates is chosen near the center at $(23,23)$ as is common in Fourier optics. Software packages often assume an origin at the lower left. This feature is accommodated conveniently for the optics point (i, j) using the coordinate transformations:

Quadrant 1 (71-i, 71-j); Quadrant 2 ( 23-i, 71-j); Quadrant 3 (23-i, 23-j); Quadrant 4 (71-i, 23-j).

The order of inputs is irrelevant to the neural network, as long as it is consistent. Each coordinate represents a tile potentially containing a particle. Hence there are $2304(48 \times 48)$ locations to be presented to the neural network. There may be multiple particles or sensors in the field of view, but the objective is to direct a trap to one tile.

The assumptions for this exercise are that only one particle or sensor appears in the $48 \times 48$ pixel field, or that only one particle or sensor appears brightly in the $48 \times 48$ pixel field, or that the filter (or lack of filter) used in zooming down shows only one sensor or particle. If the sensors or particles are too dense, then the field needs to be reduced to $24 \times 24$ or $12 \times 12$ so that only one particle or sensor is in the field. The objective is to discover the neural net resources required to generate the SLM patterns to direct traps to the tile locations. In this case, the neural net inputs consist of 0's at all locations except for a 1 at the tile location to receive the trap.

Eventually, noise will be added to the other locations to test noise immunity. The optical power in a trap is represented by the square of its magnitude. The performance of a net is evaluated by calculating the relative power in the desired trap or traps computed from the net-generated kinoforms.

The appropriate SLM pattern for a single particle is easy to calculate. The phase distribution for the kinoform for tile coordinates $(i, j)$ in the first quadrant is given by

$$
\Theta=\frac{2 \pi}{48}(\mathrm{i}-23) \mathrm{k}+\frac{2 \pi}{48}(\mathrm{j}-23) 1, \bmod 2 \pi \text {. }
$$


The phase distribution in the third quadrant is given by

$$
\Theta=\frac{2 \pi}{48}(23-\mathrm{i})(47-\mathrm{k})+\frac{2 \pi}{48}(23-\mathrm{j})(47-1), \bmod 2 \pi .
$$

Here, $\mathrm{k}$ and $\mathrm{I}$ are coordinates in the phase plot measured from $(0,0)$. Alternatively, one cycle of a kinoform algorithm ${ }^{1,12}$ can be used to generate the same result.

Figure 6 shows the phase plot for point $(27,27)$. The calculated plot is somewhat ragged as might be expected for the magnified image of the low resolution hologram. The only other requirement is that the phase used as a neural-net output be normalized in the range $[0.2,0.8]$ to accommodate the sigmoid transfer function of an output neuron of the feed-forward net. In this range, 0.2 corresponds to a phase of 0 radians and 0.8 corresponds to a phase of $2 \pi$ radians. The magnitude of the input is already correctly normalized for the feedforward net in the range $[0,1]$.

There are now 2304 possible training records containing noise-free inputs. The requirements and performance of the neural nets are easily evaluated with and without noise.

\subsection{First-stage net resources and performance}

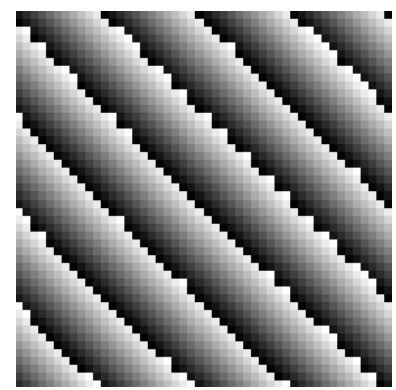

Fig. 6. Phase plot corresponding to point $(27,27)$ in $48 \times 48$ array with origin at $(0,0)$.

The feed-forward neural nets, shown schematically in Fig. 5 with a reduced number of inputs and outputs, have 2304 inputs and 2304 outputs for the first stage. Unfortunately, the nets proved to have weak generalization or interpolation capability. Consequently, the nets needed to be trained on each tile position. Another consequence was that each tile input behaved like a separate class, requiring its own hidden-layer nodes. The rule for noisy classes, discussed in the introduction, would have demanded about 6912 hiddenlayer nodes.

A rational limitation was imposed to reduce the size of the net. The 48X48-size kinoforms were required to have about 8 samples for each cycle in the range $[0,2 \pi]$. Figure 4 shows the familiar blazedgrating behavior of the phase patterns. Previous experience with the SLM showed good performance for the selection of at least 8 samples per cycle. Only the central 14X14 or 196 tile positions supported this sampling requirement. Noisy training inputs then required only about 3 X196 $=588$ hidden-layer nodes. Diagonally opposite corners of this region were bounded by coordinates $(17,17)$ and $(30,30)$.

The training performance of a net is usually measured with the root-mean-square training error as well as the response of the net to a test set that is distinct from the training set. These measures are not applicable here. The training set must contain all the tile positions, so that a distinct test set is not feasible. The overall performance is really measured by the ability of the net-generated holograms to generate traps. Performance was, therefore, defined as the ratio of the power in the intended trap to the maximum power in any extraneous traps generated by the output hologram. The input power ratio is infinite for noise-free inputs, but the output holograms for the SLM always deviate slightly from the ideal, and direct some power to other locations.

Figure 7 shows the logarithms of the training power ratios for the noise-free row-17 training records (the $x$ coordinates vary from 17 to 30 ). The net contained 196 hidden-layer nodes. Power ratios varied from 7274-to- 1 at $x=28$ to 39-to- 1 at $x=29$. There is a correlation between the rms training error and the power ratio as shown in Fig. 8. Note that power ratios are calculated by Fourier transforming the net generated holograms. 
Log Power Ratio

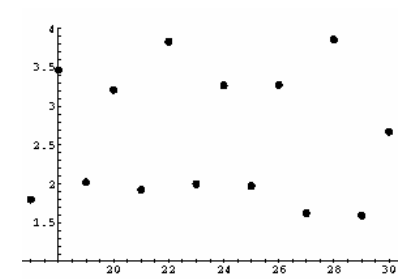

Point number in Row 17

Fig. 7. Net generated power ratios for noise-free training records.
Power Ratio

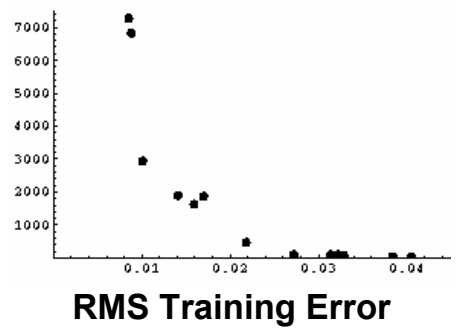

Fig. 8. Power ratio versus training error.

\section{Change in Power Ratio}

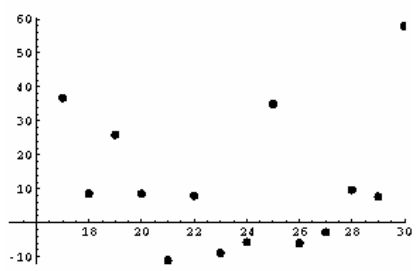

Point number in Row 17

Fig. 9. Power ratio change when noisy inputs pass through noise-free trained net.

It is a property of feed-forward nets that even deterministically trained nets will have some noise immunity. Noise inputs that had maximum powers of about 4 percent of the deterministic input were added to the training records. Input power ratios were then about 25 to 1 . Figure 9 shows the improvement in the power ratios achieved with the net-generated holograms for most of the points $(17,17)$ through $(17,30)$, when the net was tested with noisy inputs. This improvement can be made consistent for all points when the net is trained with noisy inputs.

The noise performance can be improved greatly by training with noisy inputs. A maximum noise power of 49 percent was selected, where the noise inputs were uniformly distributed between 0.0 and 0.7 . The noise inputs were generated using a double-precision random-number generator. Care must be exercised to avoid correlations in this kind of exercise. The standard rules for neural-net processing of noisy fringe patterns were used as a guide. The rules are to select uncorrelated noise sample functions equal in number to 10 percent of the number of pixels and to use 3 hidden nodes per class. These fringe-patternbased rules were not necessarily expected to apply exactly. The useful input information here was contained at one location rather than distributed over an entire pattern. In this case, the net required 3 hidden nodes per class for 588 hidden layer nodes. Noise was supplied to the full 48X48 pixel input. The NDE rule would require 231 noise sample functions per class. A rule based on the number of information containing pixels alone would require 20 noise sample functions per class. In fact, 10 sample functions per class were adequate. The net learned the 1960 training records with a rms error of 0.0127 .

The second stage requires that a net direct a trap to the correct location within a tile. Multi-pixel images of postulated particles were selected to demonstrate the second stage. For the second stage, actual particles may be large enough to cover several pixels, or may be viewed out of focus.

\section{NEURAL-NET REQUIREMENTS FOR SECOND-STAGE PROCESSING}

\subsection{Coordinates and training records}

The training records differed in three ways from the first-stage records. There were $12 \times 12=144$ coordinates that addressed actual pixel locations within a tile rather than tiles. The inputs were generated for postulated $3 \times 3=9$ pixel-size particles. Both the particles and the background can be deterministic or noisy. There were then 10X10 full-particle locations within the tile. Hence, the first and second stages together address 480X480 locations. Figure 10 shows a postulated particle magnitude distribution for a noise-free particle. Noise-distorted images were also used for training and testing. The postulated noise fluctuation range was \pm 0.35 . Figure 11 shows a noisy particle distribution. The actual particle magnitude distribution turns out not to be critical for the second stage. Finally, the background was noisy with noise power as high as 20 percent per pixel outside the particle image. The second-stage output consists of the phase distribution corresponding to a trap directed to the particle center. The inputs were normalized in the range $[0,1]$ after the noise was added. The training records then have 144 inputs and 144 outputs. The outputs are to be scaled to $480 \times 480$ pixels and added $(\bmod 2 \pi)$ to the tiled phase distributions of the first stage. 


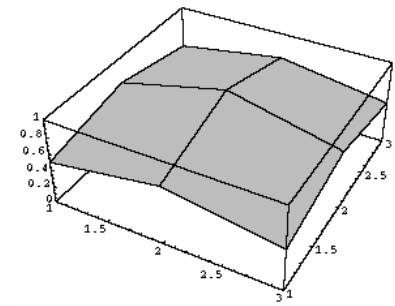

Fig. 10. Postulated scattering magnitude distribution of particle.

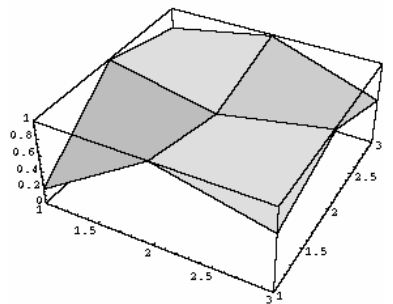

Fig. 11. Sample of noisy scattering magnitude distribution of particle.

\subsection{Second stage net resources and performance}

The old fringe-pattern-based estimate of required resources is 10 to 15 noise sample functions per pixel position and 3X144 = 432 hidden-layer nodes. In fact, a net with 432 hidden-layer nodes, and trained with a combination of deterministic particles, 15 external-noise sample functions per particle position and the 100 possible noise-free training records, did perform best for both noisy and noise-free particles. In effect, the footprints of the particles as in Figs. 10 and 11 were more important for net performance than the particles' amplitude distributions. But, a net with only 144 hidden-layer nodes performed almost as well as the net with 432 nodes.

The third stage of neural-net processing, discussed next, applies to an already-trapped particle, and is intended to detect small variations in light scattering and to provide the control information to make small changes in the trap intensity profile. By contrast, the particle intensity profile was not important for the secondstage processing already discussed in this section. The simplest implementation of the third stage is for the net output to consist of a degradable 1-of- $\mathrm{N}$ code for $\mathrm{N}$ classes. Then an output is to change gradually as the input varies from the input of the training class. The approach has been proven for fringe-pattern-based non destructive evaluation (NDE) requiring 2 classes and 2 outputs. ${ }^{10}$ The major challenge is to train the net to ignore noise sources and to respond only to small systematic changes. A systematic change is to decrease the corresponding class index, so that the net acts like a null detector for changes in the class present. A demonstration is discussed next, where the phase patterns from the first phase are used as inputs rather than outputs.

\section{DEMONSTRATION OF NEURAL-NET REQUIREMENTS FOR A THIRD-STAGE PROCESS}

\subsection{Coordinates and training records}

With three exceptions, the training records for this demonstration are simply the inverses of the $48 \times 48$ records used for the first stage. The first exception is that the inputs for training were varied with a noise source consisting of random fluctuations in the start phase. Second, the 10-percent rule was applied to the central 14X14 pixels of the original input pattern, so that there were 20 phase fluctuations per class rather than the 231 fluctuations that would normally be required by a $48 X 48$ pattern. Third, there were 196 output nodes corresponding to the 196 classes rather than 48X48 outputs representing all possible tweezers positions. These outputs constitute classification indices, and would be expected to change in response to distortions, but not noise, in the input patterns. ${ }^{10}$ Figure 6 , repeated with various start phases, would constitute examples of input records.

\subsection{An example of third-stage net resources and performance}

Training required about 3 hidden-layer nodes per class or $3 \times 196=588$ hidden layer nodes as expected. Classification outputs were qualitatively correct, and generally 0.6 or higher in the range $[0.2,0.8]$. But there were outliers. Figure 12 shows the 20 training classification outputs corresponding to point $(17,17)$. The correct class was generally selected, but the conjecture is that more noise samples per class are required for the net to learn to generate class outputs nearer to the phase-independent training index of 0.8 . The full $48 \times 48$ pattern would have required 231 rather than 20 noise samples, if the fringe-pattern rule were imposed. 
Neural Net Output

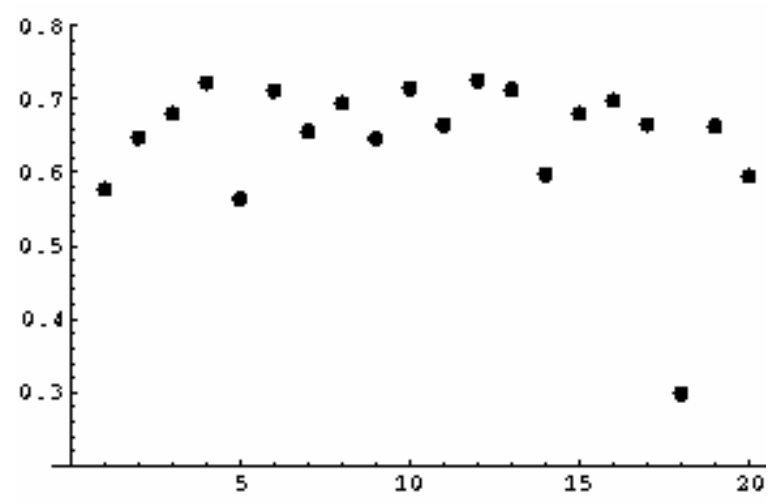

Sample Number

Fig. 12. Point $(17,17)$ classification outputs of neural net for random-phase holograms serving as neural-net inputs.

\subsection{Discussion of third-stage processing example}

Most applications are expected to require 2 to 10 classes, rather than the 196 classes used for the example. Hence most of the training resources can be devoted to training for noise immunity. The objective is to monitor the gradual change in an index as the scattering pattern is altered systematically. One exception would be a neural-net pattern-to-pattern sequencer, designed to step a tweezers trap through several profiles. Then, both input and output phase patterns might be required.

\section{CONCLUDING REMARKS}

The major conclusion is that scaling and tiling indeed allow neural nets to be adapted to controlling the full resolution of a $480 \times 480$ pixel SLM. The 3 stages of compression are able to limit the number of net inputs to less than 10,000 for each processing stage. The net designer can use previously discovered rules to estimate initially the required net resources, including the number of hidden-layer nodes and the size of the training set. Detailed design of the neural-net image-to-image controller will depend on which of a multitude of potential applications is selected. Initially, the objective is to perform simple manipulations of nanotube sensors. Stage 2 might be used to generate combined translation and rotation holograms for that application. Stage 2 might be subdivided into two stages, one for translation and one for rotation. Kinoforms are added $(\bmod 2 \pi)$ from multiple stages. Stage 3 is perhaps the most interesting stage, because it can be tested with scattering patterns generated from exact scattering theory.

\section{REFERENCES}

1. E.R. Dufresne, G.C. Spalding, M.T. Dearing, S.A. Sheets and D.G. Grier, "Computer-generated holographic optical tweezer arrays," Rev. Sci. Instrum. 72, 1810-1816 (2001).

2. A.J. Decker, "Interferometer Control of Optical Tweezers," in Interferometry XI: Applications, Wolfgan Osten, Editor, Proceedings of SPIE, Vol. 4778, 142-149 (2002).

3. R.E. Seibel, "Manipulation of Micro Scale Particles in Optical Traps Using Programmable Spatial Light Modulation," NASA/CR-2003-212726, Dec.

4. S.Y. Wrbanek and K.E. Weiland, "Optical Levitation of Micro-Scale Particles in Air," NASA/TM-2004212889, Jan.

5. A.J. Decker, "Damage Detection Using Holography and Interferometry," in Optical Metrology for Fluids, Combustion and Solids, Carolyn R. Mercer, Editor, Chapter 14, Kluwer Academic, Boston (2003). 
6. A.J. Decker and K.E. Weiland, "Sensitivity and calibration of non-destructive evaluation method that uses neural-net processing of characteristic fringe patterns," in Optical Diagnostics for Fluids, Solids, and Combustion II, Patrick V. Farrell, et. al, Editors, Proceedings of SPIE, Vol. 5191, 8-17 (2003).

7. A.J. Decker, "Self-aligning optical measurement systems," Appl. Opt. 31, 4339-4340 (1992).

8. A.J. Decker and A.E. Buggele, "Automation of Some Operations of a Wind Tunnel using Artificial Neural Networks," AIAA Journal 34, 421-423 (1996).

9. A.J. Decker, "Neural-Net Processed Electronic Holography for Rotating Machines," NASA/TM-2003212218, Feb.

10. A.J. Decker, "Optics-Only Calibration of a Neural-Net Based Optical NDE Method for Structural Health Monitoring," NASA/TM-2004-212951, Feb.

11. RSI IDL 6.0 was used for Fig. 2.

12. J.E. Curtis, B.A. Koss and D.G. Grier, "Dynamic Holographic Optical Tweezers," Opt. Comm. 207, 169175 (2002).

13. R.J. Collier, C.B. Burckhardt and L.H. Lin, Optical Holography, 560-563, Academic Press, New York (1971).

14. J.A. Lock, "Calculation of the radiation trapping force for laser tweezers by use of generalized Lorenz-Mie theory. I: Localized model description of an on-axis tightly focused laser beam with spherical aberration," Appl. Opt. 43, 2532-2544 (2004).

15. J.A. Lock, "Calculation of the radiation trapping force for laser tweezers by use of generalized Lorenz-Mie theory. II: On-axis trapping force," Appl. Opt. 43, 2545-2554 (2004).

16. B.D. Ripley, Pattern Recognition and Neural Networks, 150-155, Cambridge Univ. Press, New York (1996). 



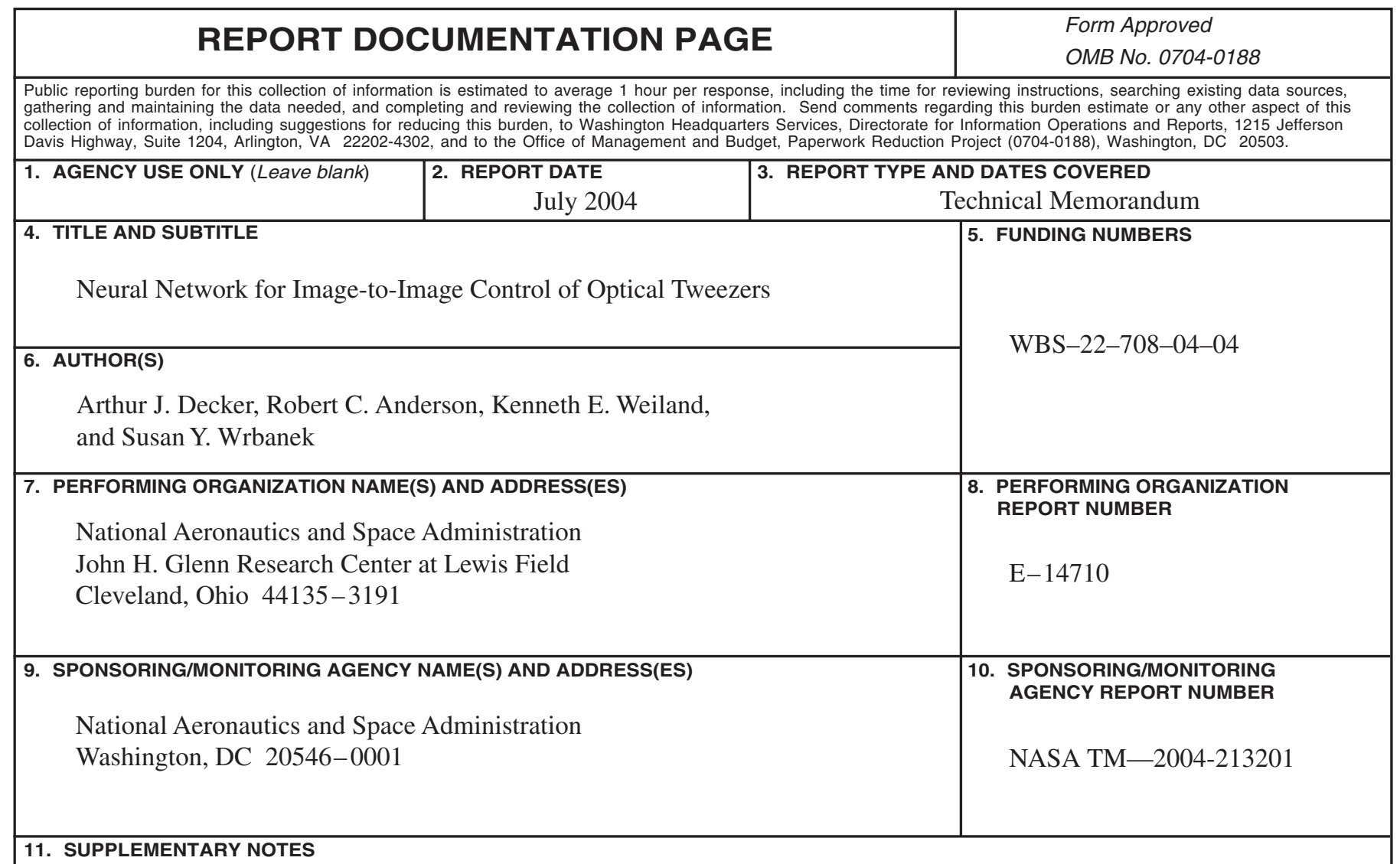

Prepared for the 2004 Annual Meeting sponsored by the International Society for Optical Engineering, Denver, Colorado, August 2-6, 2004. Responsible person, Arthur J. Decker, organization code 5520, 216-433-3639.

\begin{tabular}{|l|l}
\hline 12a. DISTRIBUTION/AVAILABILITY STATEMENT & 12b. DISTRIBUTION CODE
\end{tabular}

Restrict from public Web access based on NASA Internet Publishing Content Guidelines, November 15, 2001.

Unclassified - Unlimited

Subject Category: $35 \quad$ Distribution: Nonstandard

This publication is available from the NASA Center for AeroSpace Information, 301-621-0390.

13. ABSTRACT (Maximum 200 words)

A method is discussed for using neural networks to control optical tweezers. Neural-net outputs are combined with scaling and tiling to generate 480 by 480 -pixel control patterns for a spatial light modulator (SLM). The SLM can be combined in various ways with a microscope to create movable tweezers traps with controllable profiles. The neural nets are intended to respond to scattered light from carbon and silicon carbide nanotube sensors. The nanotube sensors are to be held by the traps for manipulation and calibration. Scaling and tiling allow the 100 by 100 -pixel maximum resolution of the neural-net software to be applied in stages to exploit the full 480 by 480 -pixel resolution of the SLM. One of these stages is intended to create sensitive null detectors for detecting variations in the scattered light from the nanotube sensors.

\begin{tabular}{|c|c|c|}
\hline $\begin{array}{l}\text { 14. SUBJECT TERMS } \\
\text { Neural networks; Image processing; Nanotechnology; Spatial light modulators; Calibration }\end{array}$ & $\begin{array}{c}\text { 19. SECURITY CLASSIFICATION } \\
\text { OF ABSTRACT } \\
\text { Unclassified }\end{array}$ \\
$\begin{array}{c}\text { 17. SECURITY CLASSIFICATION } \\
\text { OF REPORT } \\
\text { Unclassified }\end{array}$ & $\begin{array}{c}\text { 18. SECURITY CLASSIFICATION } \\
\text { OF THIS PAGE } \\
\text { Unclassified }\end{array}$ &
\end{tabular}



